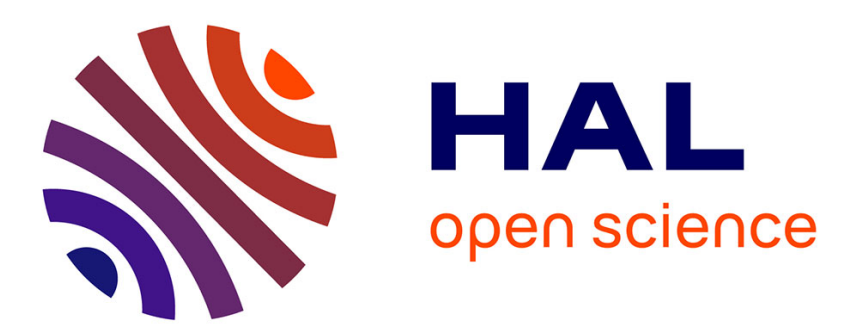

\title{
Experimental investigation of localized phenomena using Digital Image Correlation
}

\author{
Julien Réthoré, Gilles Besnard, Guillaume Vivier, François Hild, Stéphane
}

Roux

\section{- To cite this version:}

Julien Réthoré, Gilles Besnard, Guillaume Vivier, François Hild, Stéphane Roux. Experimental investigation of localized phenomena using Digital Image Correlation. Philosophical Magazine, 2008, 88 (28-29), pp.3339-3355. 10.1080/14786430802263111 . hal-00322197

\section{HAL Id: hal-00322197 https://hal.science/hal-00322197}

Submitted on 16 Sep 2008

HAL is a multi-disciplinary open access archive for the deposit and dissemination of scientific research documents, whether they are published or not. The documents may come from teaching and research institutions in France or abroad, or from public or private research centers.
L'archive ouverte pluridisciplinaire HAL, est destinée au dépôt et à la diffusion de documents scientifiques de niveau recherche, publiés ou non, émanant des établissements d'enseignement et de recherche français ou étrangers, des laboratoires publics ou privés. 


\title{
Experimental investigation of localized phenomena using Digital Image Correlation
}

\author{
J. Réthoré, ${ }^{\star}$ G. Besnard ${ }^{\sharp}$ G. Vivier, ${ }^{\sharp}$ F. Hild*,\# \& S. Roux ${ }^{\sharp}$ \\ ${ }^{\star}$ LAMCOS, INSA Lyon, 20 Avenue Albert Einstein, F-69621 Villeurbanne Cedex, France \\ \#LMT Cachan, ENS Cachan / CNRS UMR 8535 / Univ. Paris 6 / PRES UniverSud Paris \\ 61 avenue du Président Wilson, F-94235 Cachan Cedex, France
}

(v3.1 released April 2006)

\begin{abstract}
The present paper is dedicated to the use of enriched discretization schemes in the context of digital image correlation. The aim is to capture and evaluate strong or weak discontinuities of a displacement field directly from digital images. An analysis of different enrichment performances is provided. Two examples of strain localization illustrate the discussion.
\end{abstract}

\section{Introduction}

At a certain value of the macroscopic applied strain, most metallic materials experience strain localization in a small region of the tested sample. The topology and the size of this region strongly depends on the microstructure of the material [1-4]. Thus depending on the scale at which localization is modeled, various computational methods have been proposed in the literature. Among those, some do not require remeshing techniques close to the support of the localization band, such as the strong and/or embedded discontinuity methods (EDM) (see Ref. [5] for a comparative study) and extended finite element or partition of unity methods (PUM) [6-10]. For the two latter approaches, the aim is to enrich the kinematic bases of standard finite element discretizations. Strong discontinuities (i.e., when the displacement is discontinuous) [8-10] or weak discontinuities (i.e., when strain discontinuities are sought) $[11,12]$ are added to a standard finite element kinematics. Following the embedded discontinuity method, the displacement field is allowed to be discontinuous within the elements that hold additional degrees of freedom introduced by the enrichment whereas for extended finite element methods these additional unknowns are supported by the nodes of the finite element mesh.

From an experimental view point, full-field measurement techniques are used to analyse localized phenomena. Kinematic fields are evaluated by using local pattern-matching techniques (e.g., in ductile materials $[13,14]$ or brittle materials $[15,16])$. In many cases, the kinematic features of the band are not easy to capture because of the finite size of the correlation window. Temperature fields are also measured when studying, for instance, Portevin-Le Châtelier (PLC) bands [17-19]. In the latter case, global data given by strain gauges or extensometers $[20,21]$ are also used to study different types of bands.

A finite element scheme can also be used to measure a displacement field at the surface or volume of a sample using digital images that have been captured while localization occurs [22-24]. The enrichment techniques mentioned in the previous paragraph become really appealing. When dealing with images discretized by a set of square pixels, the most appropriate way to generate a finite element mesh is to use square elements aligned with the direction of the image pixel map (element edges usually do not cut pixels). The ability of EDM or PUM to account for a discontinuity whose support is not described by the underlying mesh is essential. Using these enrichment techniques, one may have access to quantitative measurements of displacement or strain discontinuities directly from digital images.

Concerning strong discontinuities, PUM have been applied directly in an experimental context $[25,26]$. As

\footnotetext{
${ }^{*}$ Corresponding author. Email: hild@lmt.ens-cachan.fr

Philosophical Magazine

ISSN 1478-6435 print/ISSN 1478-6443 online (c) 200x Taylor \& Francis

http://www.tandf.co.uk/journals

DOI: 10.1080/1478643YYxxxxxxxx
} 
will be shown in the sequel, tracking weak discontinuities cannot be performed from a direct application of any "standard" enrichment technique. After an introduction to digital image correlation and PUM, a noise sensitivity analysis and an uncertainty study for strong and weak discontinuous enrichments are carried out. A new "reduced" weak discontinuous enrichment is subsequently proposed. Then, two examples are presented. The first one is dedicated to the analysis of a shear band that is described by a strong discontinuity approach, while the second one deals with Portevin-Le Châtelier bands that are captured using a weak discontinuity approach.

\section{Digital image correlation}

The principe of digital image correlation is to find a displacement field $\mathbf{u}$ that satisfies the optical flow conservation between two digital images. $f$ being the reference image (a grey level valued discrete function), and $g$ the deformed image, the problem is recast as the minimization of the following local residual

$$
\Phi^{2}(\mathbf{x})=(f(\mathbf{x})-g(\mathbf{x}-\mathbf{u}(\mathbf{x})))^{2}
$$

The minimization of $\Phi^{2}$ is an ill-posed problem (one scalar equation for one unknown vector). Furthermore, it is highly non-linear and the image functions $f$ and $g$ are generally noisy. To circumvent those difficulties, a global linearized formulation is chosen. It consists in integrating $\Phi^{2}$ over the domain of interest $\Omega$

$$
\eta^{2}=\iint_{\Omega}\left[g\left(\mathbf{x}-\mathbf{u}^{i}\right)+\mathrm{d} \mathbf{u} \cdot \nabla g\left(\mathbf{x}-\mathbf{u}^{i}\right)-f(\mathbf{x})\right]^{2} \mathrm{~d} \mathbf{x}
$$

where superscripts denote the iteration index of the Newton procedure, and $g\left(\mathbf{x}-\mathbf{u}^{i+1}\right)$ has been linearized by assuming that the increment du between iterations $i+1$ and $i$ is small. By adopting a discretization scheme

$$
\mathbf{u}(\mathbf{x})=\sum_{n \in \mathcal{N}} a_{n} \psi_{n}(\mathbf{x})=[\mathbf{\Psi}(\mathbf{x})]\{\mathbf{U}\}
$$

where $\boldsymbol{\psi}_{n}$ holds for the $n^{\text {th }}$ interpolation function and $a_{n}$ its associated degree of freedom, the resolution of the problem is carried out iteratively through a series of linear systems

$$
[\mathbf{M}]^{i}\{\mathrm{~d} \mathbf{U}\}=\{\mathbf{b}\}^{i}
$$

with

$$
M_{n m}^{i}=\iint_{\Omega}\left(\boldsymbol{\psi}_{n} \cdot \nabla g\left(\mathbf{x}+[\mathbf{\Psi}]\{\mathbf{U}\}^{i}\right)\right)\left(\boldsymbol{\psi}_{m} \cdot \nabla g\left(\mathbf{x}+[\mathbf{\Psi}]\{\mathbf{U}\}^{i}\right)\right) \mathrm{d} \mathbf{x}
$$

and

$$
b_{n}^{i}=\iint_{\Omega}\left(\boldsymbol{\psi}_{n} \cdot \nabla g\left(\mathbf{x}+[\mathbf{\Psi}]\{\mathbf{U}\}^{i}\right)\right)\left(f(\mathbf{x})-g\left(\mathbf{x}+[\mathbf{\Psi}]\{\mathbf{U}\}^{i}\right)\right) \mathrm{d} \mathbf{x}
$$

The gradient of the image is computed by finite differences and the sub-pixel interpolation of the grey levels is cubic spline. At this point, the basis functions $\psi$ can be chosen arbitrarily. Since the image is naturally partitioned into pixels, it is appropriate to choose a square or rectangular shape for each correlation element. This leads us to the choice of Q4-finite elements as the simplest basis. Each element is mapped onto the square $[0,1]^{2}$, where the four basic functions are $(1-x)(1-y), x(1-y),(1-x) y$ and $x y$ in a local $(x, y)$ frame. The technique will therefore be referred to as Q4 Digital Image Correlation (Q4-DIC). 


\section{Partition of unity method}

The partition of unity property of finite element shape functions $\left(N_{n}\right)_{n \in \mathcal{N}}$ reads

$$
\sum_{n \in \mathcal{N}} N_{n}(\mathbf{x})=1
$$

where $\mathcal{N}$ is the set of nodes inside the domain of interest and $N_{n}$ their associated finite element shape functions. This property is used to enrich the standard finite element approximations [6] in the following way

$$
\mathbf{u}(\mathbf{x})=\sum_{j=1,2} \sum_{n \in \mathcal{N}} a_{n j} N_{n}(\mathbf{x}) \mathbf{X}_{j}+\sum_{j=1,2} \sum_{n \in \mathcal{N}_{e}} d_{n j} N_{n}(\mathbf{x}) F(\mathbf{x}) \mathbf{X}_{j}
$$

where $\mathbf{X}_{j}$ are the basis vectors of the image plane, $a_{n j}$ standard degrees of freedom, $F$ the enrichment function and $d_{n j}$ its associated degrees of freedom that are supported by the set of nodes $\mathcal{N}_{e}$. Let us note that, thanks to Equation (7), the enrichment function $F$ is evaluated exactly by the discretization scheme. The function $F$ has to be chosen in order to capture the pre-assumed solution of the problem. For example, one should use a Heaviside function for strong discontinuity capturing or a distance function when a weak discontinuity is to be sought.

In practice, a discontinuous enrichment is obtained using the following function [27]

$$
H_{n}(\mathbf{x})=\mathcal{H}(\mathbf{x})-\mathcal{H}\left(\mathbf{x}_{n}\right)
$$

whereas the weak discontinuity enrichment reads

$$
\tilde{\phi}(\mathbf{x})=\phi(\mathbf{x})-\sum_{n \in \mathcal{N}} N_{n}(\mathbf{x}) \phi\left(\mathbf{x}_{n}\right)
$$

where $\phi$ is the distance to the support of the discontinuity. This procedure extracts from the initial enrichment $(\mathcal{H}$ and $\phi)$ the only contribution that cannot be captured by finite element shape functions. Thus, a better conditioning of the problem is obtained (for a detailed discussion, see Ref. [12]).

Within the present framework, the technique consists of an eXtended Digital Image correlation (X-DIC) approach. Coupled with a Q4 discretization, it will be called X-Q4. The enrichment strategies presented above require the knowledge of the geometry of the support of the discontinuity for both strong and weak discontinuities. As shown in Ref. [25], the residual map $\Phi^{2}(\mathbf{x})$ is a good indicator to use for setting the location of the discontinuity properly. In practice, a first displacement measurement is performed by using a standard Q4 discretization. Examining the residual map $\Phi^{2}(\mathbf{x})$ resulting from this calculation reveals the quality for the displacement estimation in general, but also regions where the continuous Q4 kinematics fails at representing the actual displacement field. Hence, strong discontinuities (e.g., cracks or shear bands) are detected when using the residual map. An optimization of the discontinuity shape is proposed in Ref. [25]. Weak discontinuities are not as visible on the residual map as strong discontinuities, but again an error criterion seems to be appropriate as will be demonstrated in Section 6 .

\section{A priori performances and noise sensitivity analysis}

Since the correlation of two images is an ill-posed problem, one must pay attention to noise sensitivity, measurement error and uncertainty. For the two latter points, the performance of the correlation algorithm may be qualified by artificially deforming an image and evaluating the difference between the prescribed motion and the measured solution. 


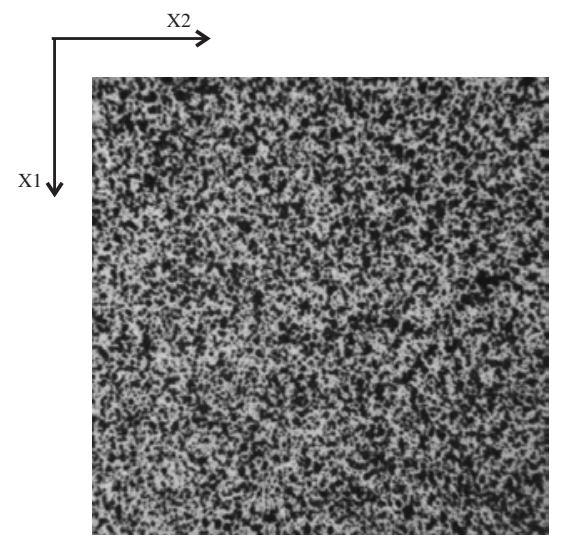

$-a-$

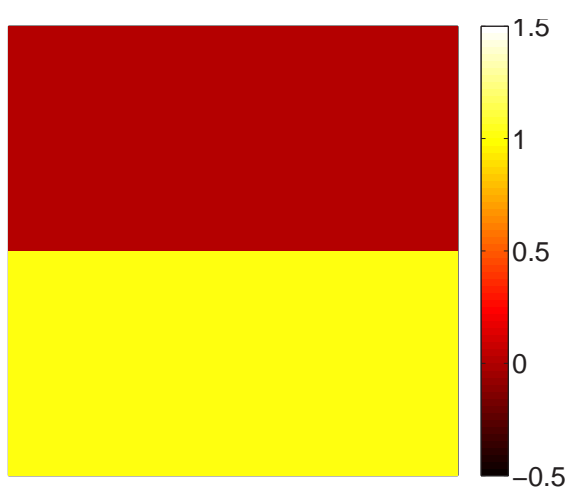

-b-

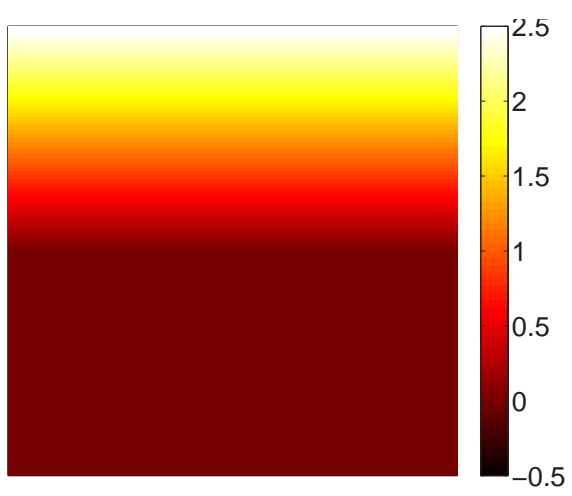

$-\mathrm{C}-$

Figure 1. Reference picture for the noise sensitivity analysis (a), horizontal prescribed displacement for the strong discontinuity analysis (b) and vertical prescribed displacement for the weak discontinuity analysis (c).
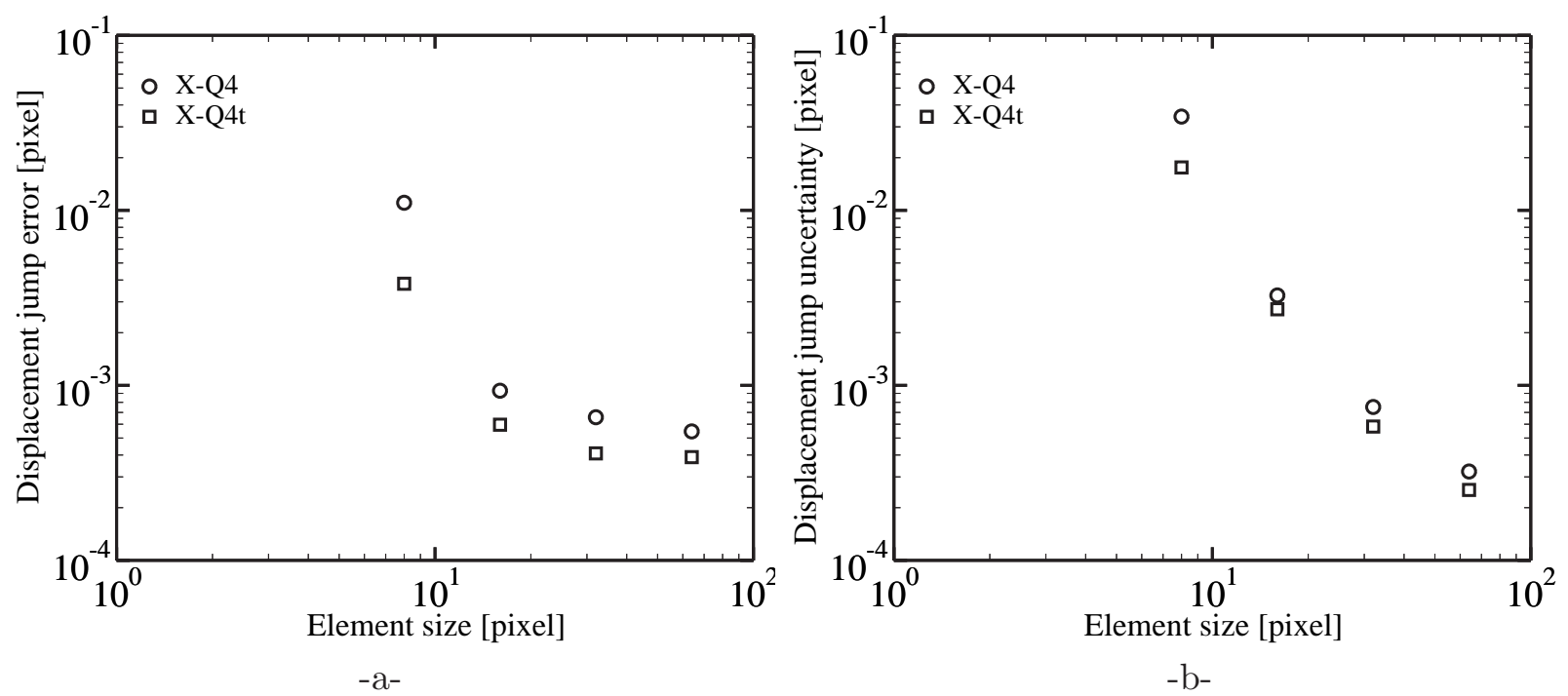

Figure 2. Displacement discontinuity error and standard uncertainty as functions of the element size (in pixels) computed with different algorithms: X-Q4 corresponds to a classical X-FEM discretization with a discontinuous enrichment and X-Q4t for a discretization with a discontinuous enrichment in the tangential direction only.

\subsection{Strong discontinuity}

First, a discontinuous translation in the tangential direction is applied to a reference image (Figure 1a). After performing the correlation analysis, the computed displacement jump along the discontinuity line is compared to the prescribed value. This artificial motion is assumed to model strain localization observed at a scale for which the band thickness is vanishingly small (Figure 1b).

Figure 2 shows the error and standard uncertainty of the displacement jump estimation obtained for different element sizes. The error is defined as the absolute value of the average difference between the estimated and prescribed displacement jump, and the standard uncertainty is the corresponding standard deviation. The results in Figure 2 are mean values for a described displacement jump ranging from 0 to 1 pixel. One observes that a rapid decrease of the error and uncertainty is obtained when the element size is increasing. In this case, the number of degrees of freedom per unit area of image is decreasing and the solution is better constrained. Error and uncertainty follow the same trend, and levels as low as $10^{-3}$ pixel are reached.

In Figure 2, results obtained with a discretization that involves enriched degrees of freedom only for the component of the displacement in the tangential direction to the band (X-Q4t) are also reported. The total number of enriched degrees of freedom is then reduced by a factor 2 with respect to an enrichment in both directions (X-Q4). Consequently, they are better constrained than in the previous case and the 

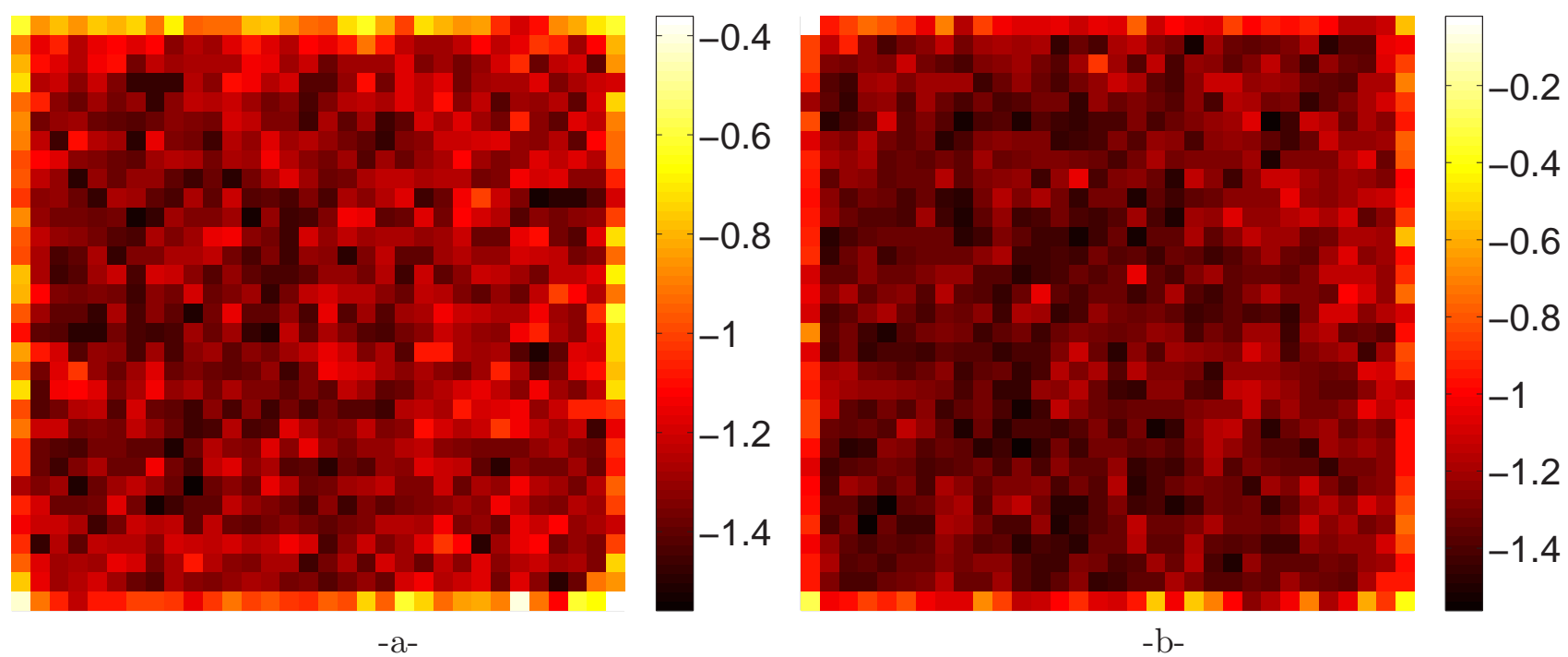

Figure 3. Sensitivity map in pixel/grey level of the degrees of freedom in the vertical (a) and horizontal (b) directions for a standard (Q4) finite element discretization in decimal log-scale.
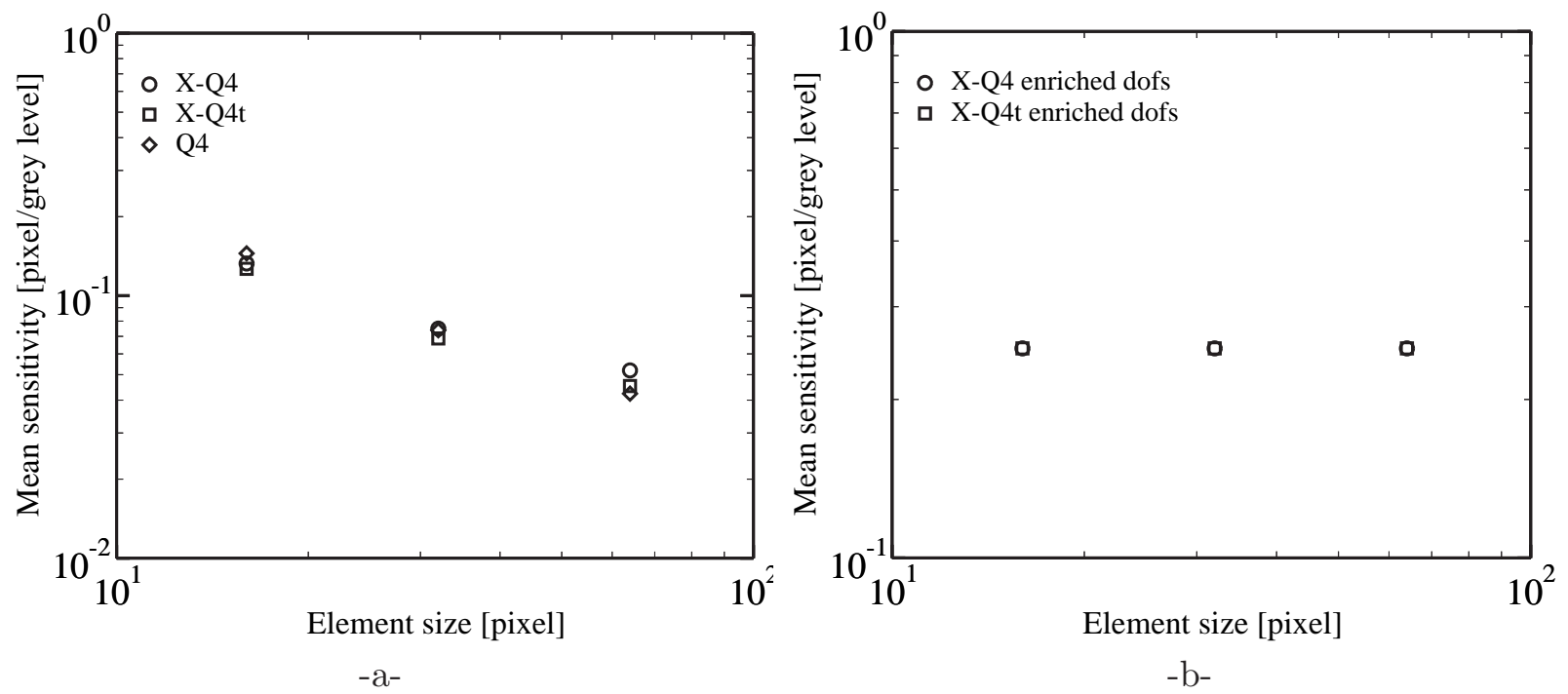

Figure 4. Mean sensitivity (a) and mean sensitivity for the extended dofs (b) as functions of the element size (in pixels) computed with different algorithms: Q4 corresponds to a FEM discretization, X-Q4 to a classical X-FEM discretization with a discontinuous enrichment and X-Q4t for for an enrichment in the tangential direction only.

performances are enhanced, namely, error and uncertainty are (approximately) reduced by a factor of 2 .

Another point of view can be adopted after a perturbation analysis of Equation (4) is carried out. Let us consider that the reference $f$ and deformed $g$ pictures are corrupted by a (random) noise. This noise is supposed be of zero mean and spatially uncorrelated. The deformed image $g$ can be conventionally considered as noiseless and the deviation from optical flow conservation allows one to define an effective noise, which only affects the reference image $f$. Thus, the variance of this effective noise is twice the variance of the initial noise $2 \sigma^{2}$. Examining Equation (4) and the definition of $[\mathbf{M}]$ and $\{\mathbf{b}\}$, the perturbation induced on the displacement vector $\{\delta \mathbf{U}\}$ is of zero mean but is spatially correlated. Its correlation kernel $[\mathbf{C}]$ is given by

$$
\left\langle\{\delta \mathbf{U}\}\{\delta \mathbf{U}\}^{T}\right\rangle=2[\mathbf{C}] \sigma^{2}=2[\mathbf{M}]^{-1} \sigma^{2}
$$

Figure 3 gives the square root of the diagonal elements of $\mathbf{C}$, i.e., the expected standard deviation, for the displacement unknowns in the vertical or horizontal directions. These sensitivity maps are not homogeneous due to the heterogeneous nature of the gradient of the reference image. One notes that the 

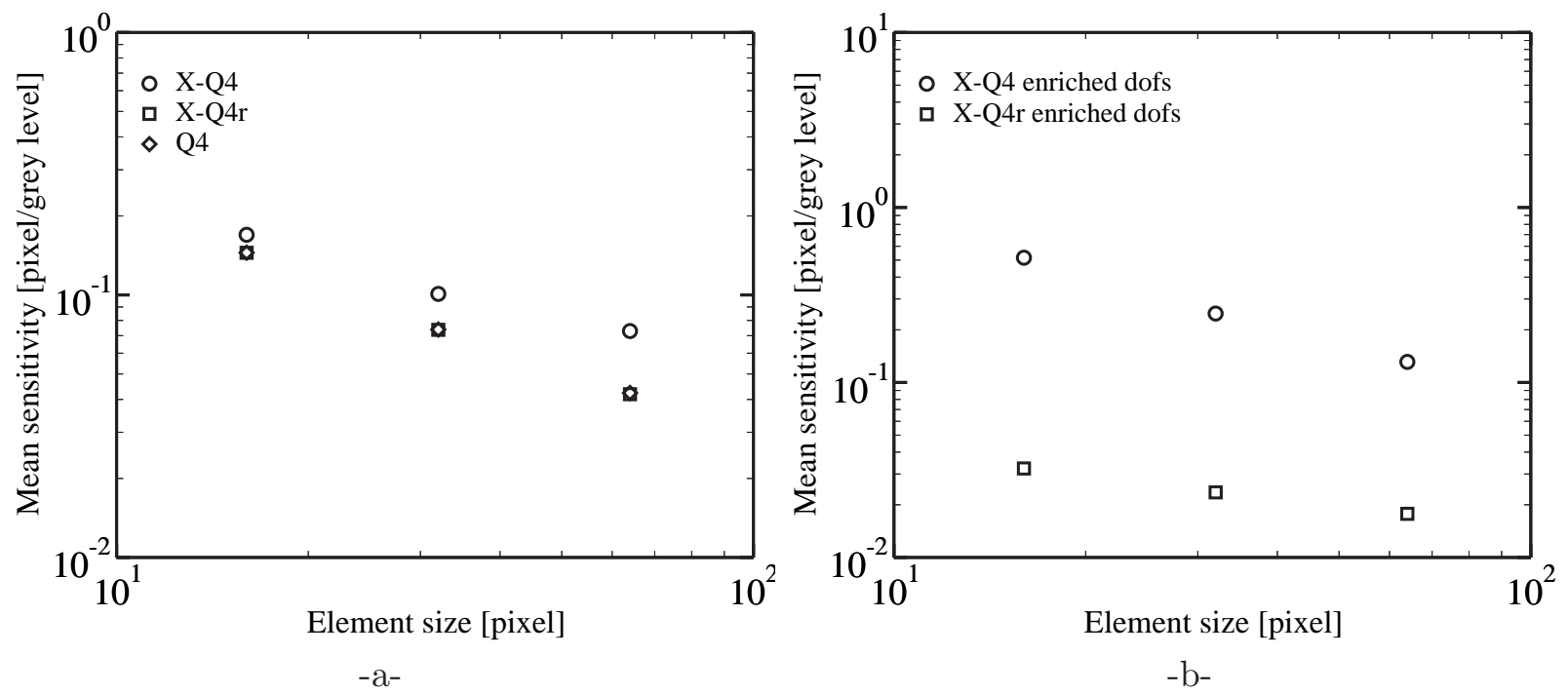

Figure 5. Mean sensitivity (a) and mean sensitivity for the extended dofs (b) as functions of the element size (in pixels) computed with different algorithms: Q4 corresponds to an FEM discretization, X-Q4 to a classical X-FEM discretization with a weakly discontinuous enrichment and $\mathrm{X}-\mathrm{Q} 4 \mathrm{r}$ for its regularized version.

degrees of freedom held by the nodes on the boundary are much more sensitive than those in the bulk, and even more so for those held by the corner nodes. The support of those nodes (i.e., the elements that have those nodes in their connectivity) are one half — or one quarter for corner nodes - the support of the nodes in the bulk. This approach reveals to be an appropriate indicator for evaluating the conditioning of the enriched degrees of freedom of PUM applied to digital image correlation. Figure 4 shows the change of the noise sensitivity averaged over all the degrees of freedom for a standard approach (Q4), an enriched approach (X-Q4) and the X-Q4t approach. One observes that the noise sensitivity is decreasing when the element size increases and also that the X-Q4t approach gives a lower noise sensitivity than the X-Q4 approach. Figure 4b presents the noise sensitivity averaged over the enriched degrees of freedom only. The two enriched approaches give the same averaged "enriched" noise sensitivity but as X-Q4 invokes twice more enriched degrees of freedom, the average noise sensitivity of this approach is higher. These results show the beneficial effect of a coarse discretization allowed by extended techniques in the framework of digital image correlation.

\subsection{Weak discontinuity}

Let us now perform the same analysis for a weak discontinuity. Even if the regular part of the enrichment function has been extracted (see Equation (10)), this enrichment is of the same polynomial order as the standard finite element part of the discretization and a very high noise sensitivity is expected. Figure 5 shows the comparison of the mean noise sensitivity for the enriched and standard Q4 approaches and a dramatic increase of the noise sensitivity is obtained for the enriched discretization. To circumvent this lower confidence level, a regularized approach (X-Q4r) is proposed. It consists in prescribing the same value to all the enriched degrees of freedom. Therefore, the regularized approach involves only one enriched unknown for each direction. These two enriched degrees of freedom are not associated to nodes but to the set of elements that are cut by the discontinuity line. In this sense, the proposed approach may also be considered as a regularized EDM where the continuity of the discontinuity patterns were enforced. As shown in Figure 5, the proposed regularization allows for a noise sensitivity as low as that of the standard Q4 approach. Furthermore, the noise sensitivity of the enriched degrees of freedom is divided by a factor of 10 when compared with the X-Q4 approach.

Figure 6 reports the results of the error and uncertainty analyses obtained by artificially stretching the reference image with a weakly discontinuous displacement map (for a strain discontinuity ranging from 0 and 0.001) as shown in Figure 1c. Figure 6 confirms the higher robustness of the regularized approach. It is worth noting that the uncertainty of the regularized approach is very low (down to the numerical 

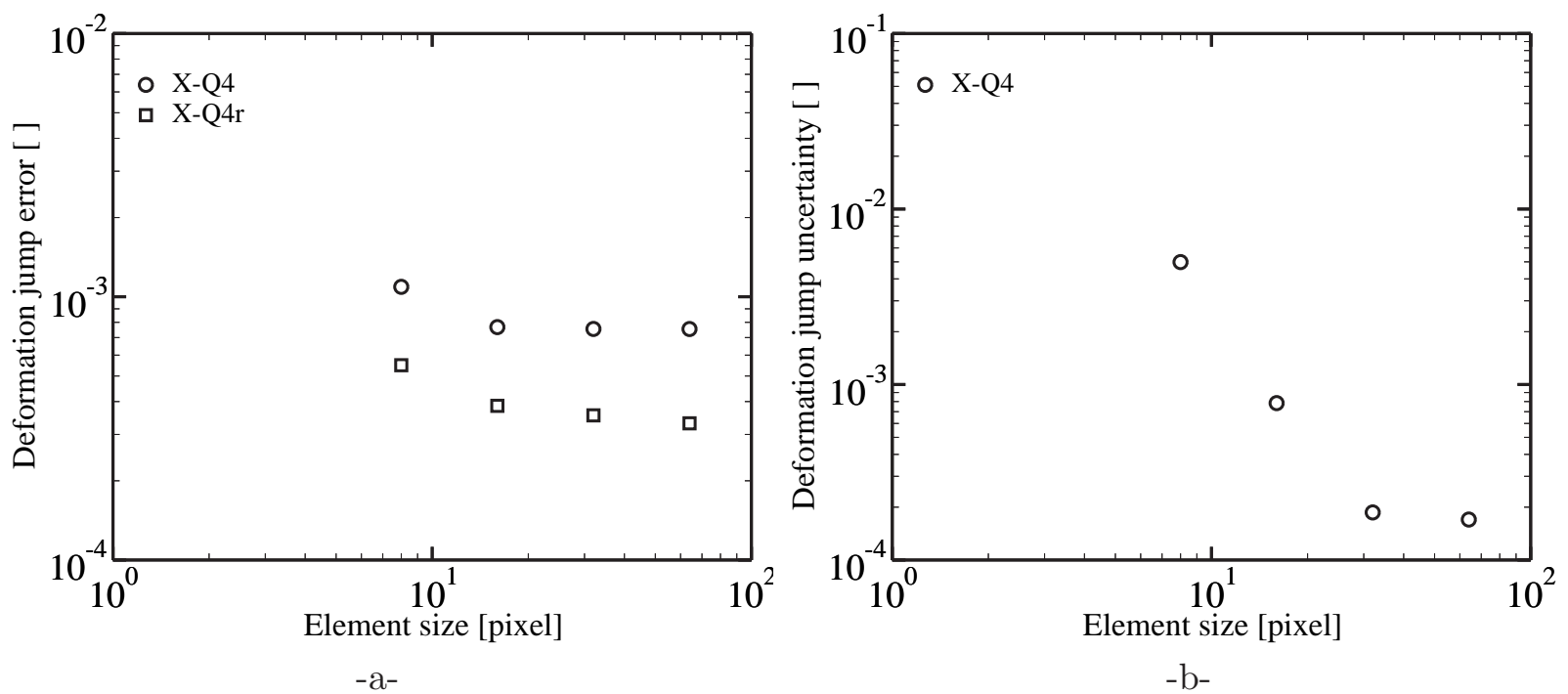

Figure 6. Strain discontinuity error and uncertainty as a function of the element size (in pixels) computed with different algorithms: $\mathrm{X}$-Q4 corresponds to a classical X-FEM discretization with a weakly discontinuous enrichment and X-Q4r for its regularized version.

limit) because the strain discontinuity that is allowed by the discretization scheme remains constant over the entire set of elements cut by the discontinuity line. Furthermore, the error level obtained with the regularized approach is 5 times less than that obtained with the standard enriched X-Q4 scheme.

The extension of the digital image correlation technique to enriched functions appears as straightforward technically. However, for some cases (such as weak discontinuities) this enrichment naturally leads to a poor numerical conditioning, and hence, even though the displacement basis has been enriched to capture a feature of interest, its determination may be worthless because too uncertain. It is thus absolutely essential to rely on the type of presented analysis to get a proper evaluation of the real error, uncertainty and noise sensitivity. Let us also underline the fact that if the searched feature is now included in the displacement basis, much larger meshes may be used, hence leading to a more accurate and less uncertain determination of the displacement. Last, if the enriched basis still remains poorly conditioned, then a regularization may be used, and again the noise sensitivity analysis is a useful tool to choose the amount of regularization that is required. In the X-Q4 approach, the strain discontinuity is described by the shape functions intersected by the support line of the discontinuity, whereas for X-Q4r the discontinuity is a constant. Intermediate regularizations could have been considered with a few degrees of freedom to describe the discontinuity variation along its support. This strategy is valid irrespective of the chosen type of enrichment.

In the present analyses, the discontinuity in the mesh is positioned so that the elements are cut in the middle of their edges. For real cases, the position of the element with respect to the discontinuity is arbitrary. The method behaves in the same manner as X-FEM behaves in the context of mechanical analysis, namely, ill-conditioning is obtained when the discontinuity is close to a node (with the modified discontinuous enrichment function used herein) and it is artificially moved so that the two parts of the corresponding element are not too large/small. This is usual in the context of a mechanical analysis. Here, the main difference is that, due to the discretization of the image in pixels, the minimum distance between the crack and a node is 1 pixel. Hence, a natural limit value of the above/below ratio is obtained.

Last, let us note that there are two independent issues, namely, the identification of the geometry of the band (shape and position) and the displacement measurement. The performance analysis of the digital image correlation algorithm presented herein does not account for the geometrical identification of the band. For a complex band geometry, the performance may decrease but there are as many band geometries as tested samples. That is the reason why the performance analysis is only carried out for an idealized band shape. Let us now consider the application of these techniques to two experimental cases of strain localization (one strong and one weak discontinuities). In the second case, the identification of the geometry of the band will be discussed. 

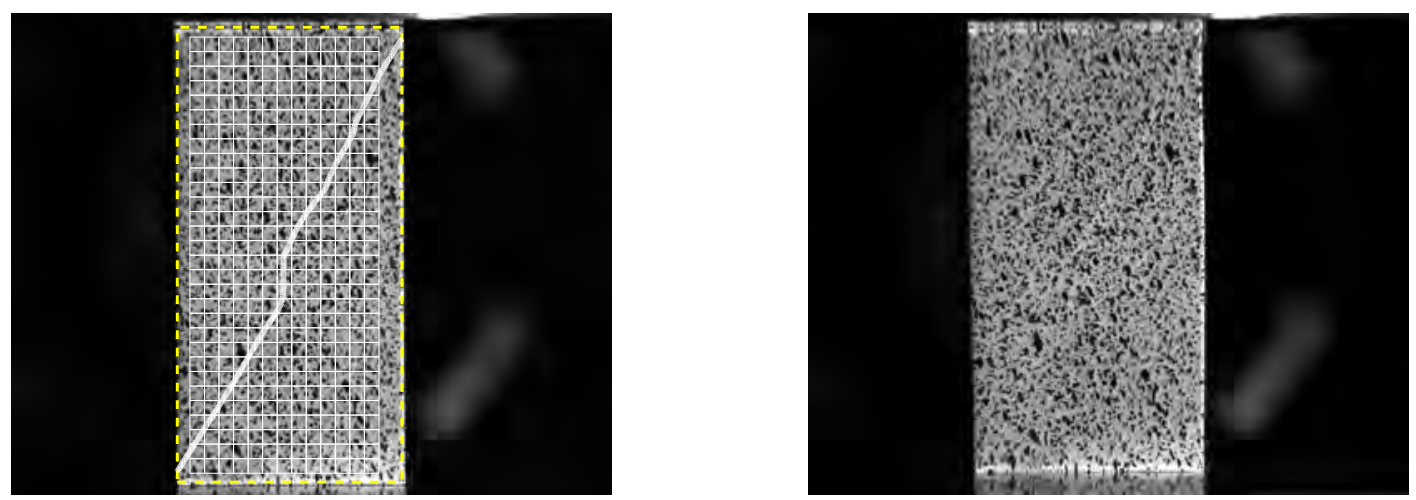

Figure 7. Region of interest, mesh, detected shear band and the deformed picture.

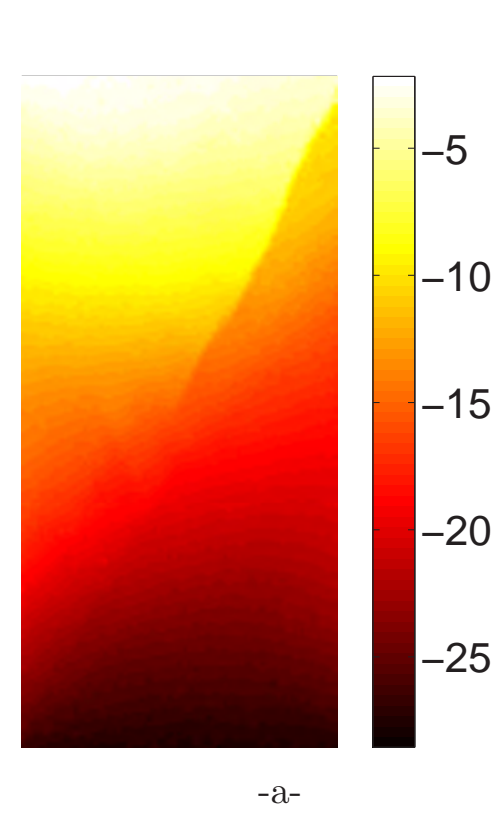

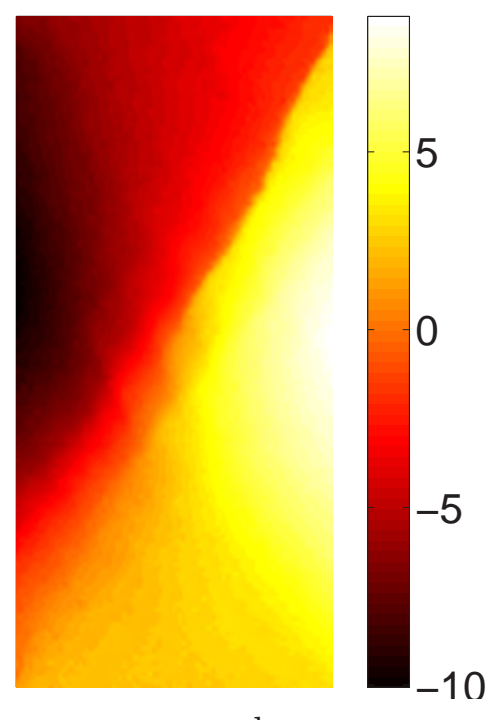

$-\mathrm{b}-$

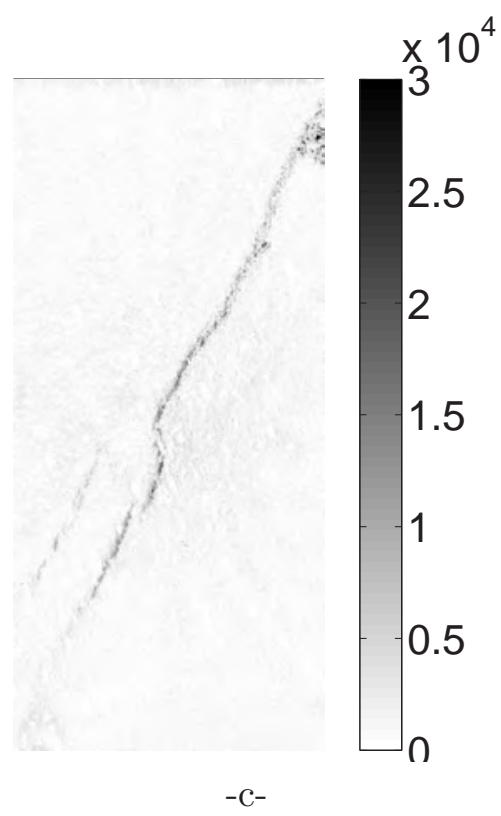

$-\mathrm{C}-$

Figure 8. Vertical (a) and horizontal (b) displacements obtained by using a standard finite element discretization (Q4) with 8-pixel elements and the corresponding error (c) map that is used to define the geometrical support of the band.

\section{Compression test on an HMX-based energetic material}

Figure 7 shows two pictures of a pressed HMX-based energetic material subjected to a compression test. It is a concrete-like material, containing large $(200-630 \mu \mathrm{m})$ HMX grains (i.e., the aggregates) embedded in a composite matrix (the mortar) itself containing small $(0-100 \mu \mathrm{m})$ HMX grains, a few percents of a thermoset binder, and around 1\% porosity [28]. Figure 8 shows the displacement map that was obtained by using a Q4 discretization with 8-pixel elements in the correlation algorithm. Exploring these displacement maps reveals a very thin localization zone. The error map on Figure 8-c appears to be a good indicator of the localization band. One may note that at the scale of the picture (i.e., the physical size of 1 pixel is $50 \mu \mathrm{m})$, the shear-band appears as a line. If a smaller scale had been reached by the observation setup, a finite band width may have been revealed. In the present case, a discontinuous enrichment is adopted along the line where maximum error levels are obtained (Figure 7).

The results presented in Figure 9 are obtained by using the first proposed extended digital image correlation technique (X-Q4), namely, strain localization is captured in the detected interface whereas Q4-DIC only provides a smoothed description of this phenomenon. If a more accurate description of the localization interface were required with Q4 elements, smaller elements should have been used, yet leading 

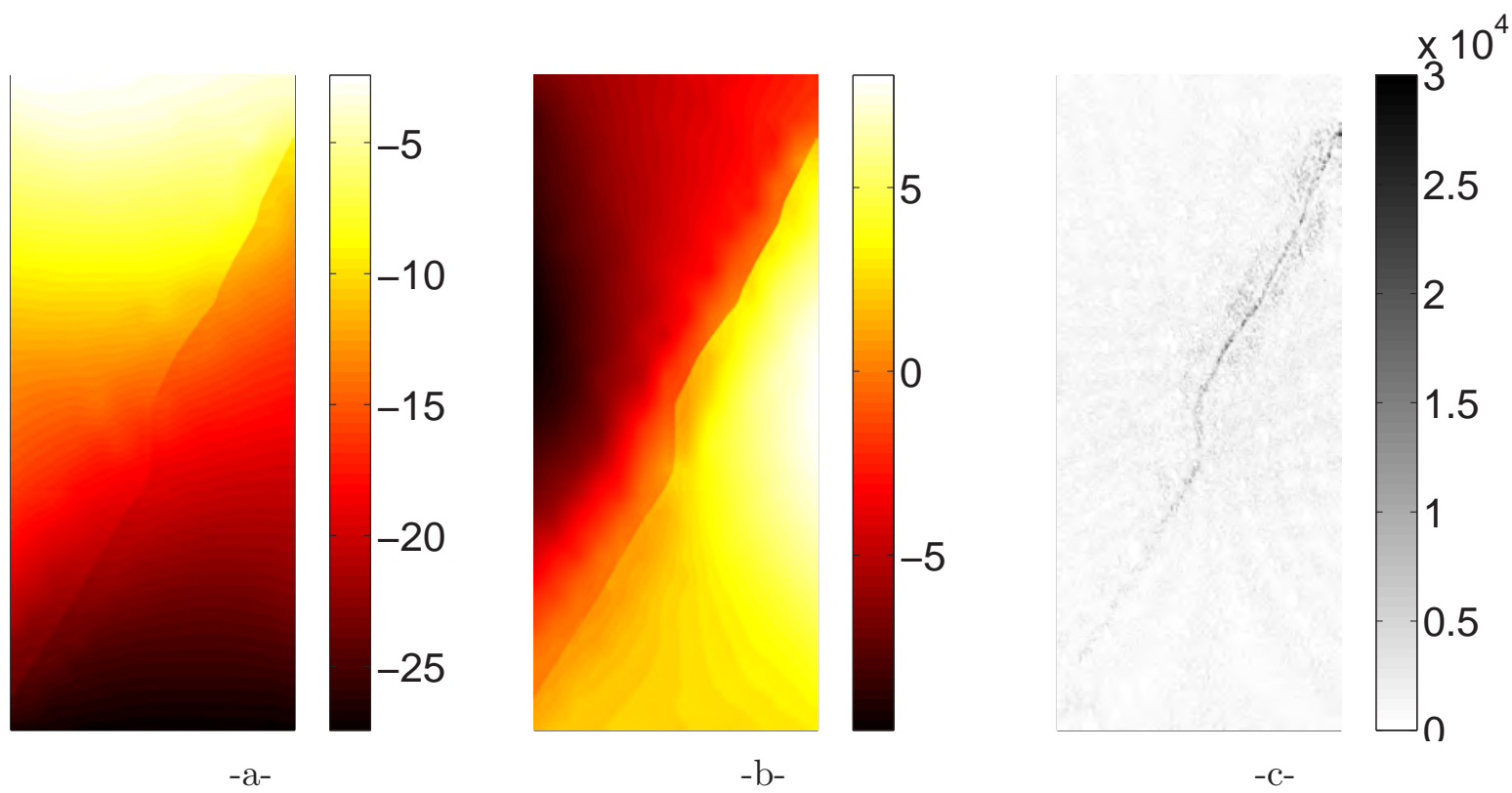

Figure 9. Vertical (a), horizontal (b) displacement map (in pixels) and error map in grey level using 32-pixel elements with discontinuous enrichments.

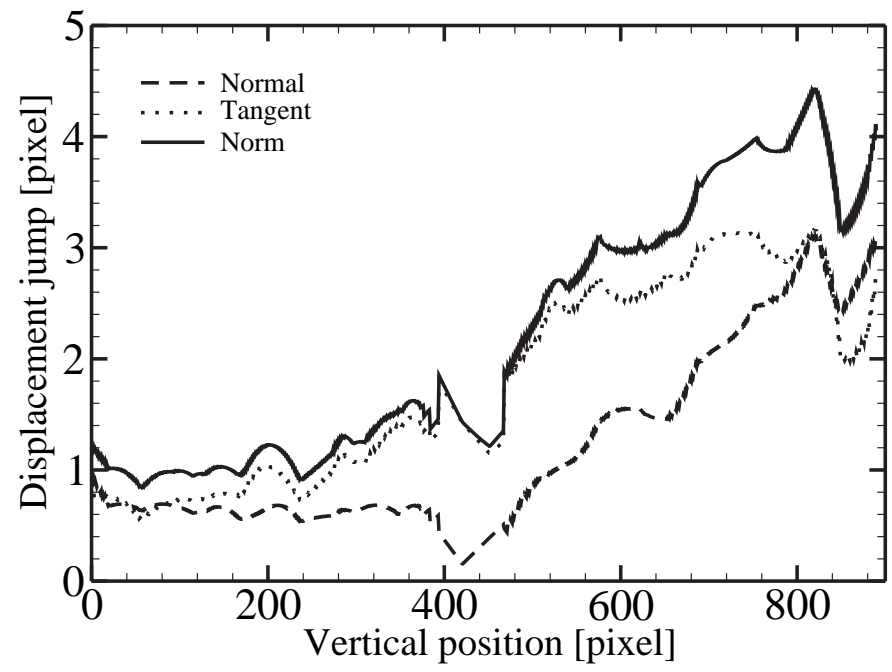

Figure 10. Displacement jumps for 32-pixel elements.

to higher uncertainty levels. In this experimental case, the benefit of using enriched finite elements in the context of digital image correlation is shown since it allows for the description of localized phenomena with coarser meshes, i.e., with lower displacement uncertainties.

Another benefit is that the discontinuity of the displacement is measured experimentally as shown in Figure 10. It is to be noted that the band first develops from the upper right corner of the region of interest where the displacement discontinuity is higher (greater than 4 pixels in norm). The tangent contribution to the displacement jump is almost everywhere greater than the normal one. Concerning the normal displacement jump, dilatancy effects are captured, allowing for the estimation of the band width. Let us note that the fact that only one face view is examined (whereas a discontinuity of the component of the displacement normal to the observation plane is also expected) may induce an apparent normal discontinuity in the plane in addition to actual dilatancy effects. The apparent normal discontinuity would be expected ideally to be the projection of the 3D tangential discontinuity onto the observation plane. This point lies beyond the scope of the present paper. 


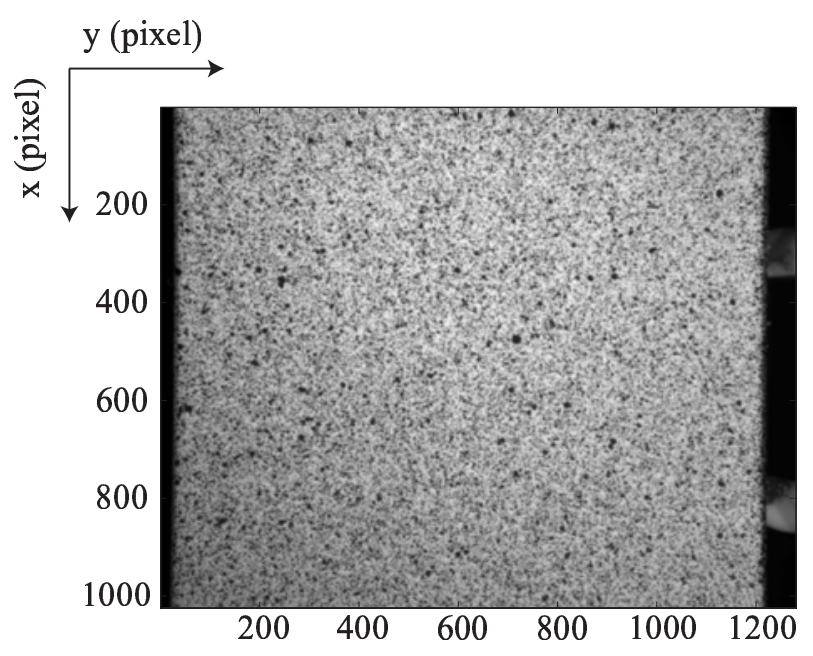

Figure 11. Reference picture for the Portevin-Le Châtelier bands.

\section{Portevin-Le Châtelier band}

The second example concerns an aluminum alloy of type 5005. The sample is coated with sprayed black and white paints to create the random texture for the displacement field measurement (Figure 11). In the present case, the physical size of one pixel is $25 \mu \mathrm{m}$. A controlled displacement rate of $10 \mu \mathrm{m} / \mathrm{s}$ is applied to study strain localization and pictures are taken for $60 \mu \mathrm{m}$-increments. The following analysis of the displacement field is an increment between two image acquisitions in the "plastic" regime.

To achieve a good spatial resolution with a standard Q4 approach, 16-pixel elements are used. Figure 12 shows that the displacements are reasonably captured. The strain levels become too small to have a trustworthy evaluation. However, a band of high strain level is observed. The mean longitudinal strain within the band is about 0.005 whereas it is an order of magnitude lower in the other parts. To obtain a more accurate description of the strain discontinuity across the limit of the band, one may wish to reduce the size of the elements. It was shown that for very small element sizes (such as 4 to 8 pixels) "integer locking" (i.e., the tendency for displacements that are close to half-integer values to stick to the closest integer) appears and produces an artificial enhancement of the strain right on the limit part of the band [22]. Thus, mesh refinement does not provide a better description of strain localization, and an alternative solution is desirable.

The regularized enriched approach, X-Q4r, is used hereafter. The element size is increased eight times in both directions so that much lower uncertainty levels are obtained. The results of the analysis are shown in Figure 13 and compared to those obtained with the standard Q4 approach. In the following, only two morphological parameters are considered to describe the band, namely, an angle and a width. This hypothesis can be relaxed at the expense of larger measurement uncertainties. The compromise made here was to favor the uncertainty level of the measured enriched degrees of freedom. The position, width and angle of the band are evaluated using a minimization of the mean value of the correlation residuals $|\Phi|$. Figure 15 shows this error indicator as a function of the angle, the vertical shift and the width of the band. The optimal band geometry is given by a vertical shift of 311 pixels with respect to the middle top point of the region of interest, a width of 248 pixels and an angle of $65^{\circ}$. Using this optimal geometry, the mean error is 922.3 grey levels to be compared to the dynamic range of the reference image over the region of interest of 40416 grey levels. The strain discontinuity is evaluated as 0.0035 for the normal strain $\varepsilon_{n n}$ and 0.0026 for the shear strain $\varepsilon_{t n}$ at the upper boundary of the band, whereas we obtain -0.0035 and -0.0027 respectively for the lower boundary. Note that the tangent strain $\varepsilon_{t t}$ is continuous (Figure 13). Figure 14 proposes a comparison between the residual maps obtained with the optimal geometry of the $\mathrm{X}$-Q4r approach and a standard Q4 approach. The latter reveals similar error levels with a mean value of 922 grey levels over the region of interest for pictures with a dynamic range of 16 bits. This result validates the approach proposed herein. 

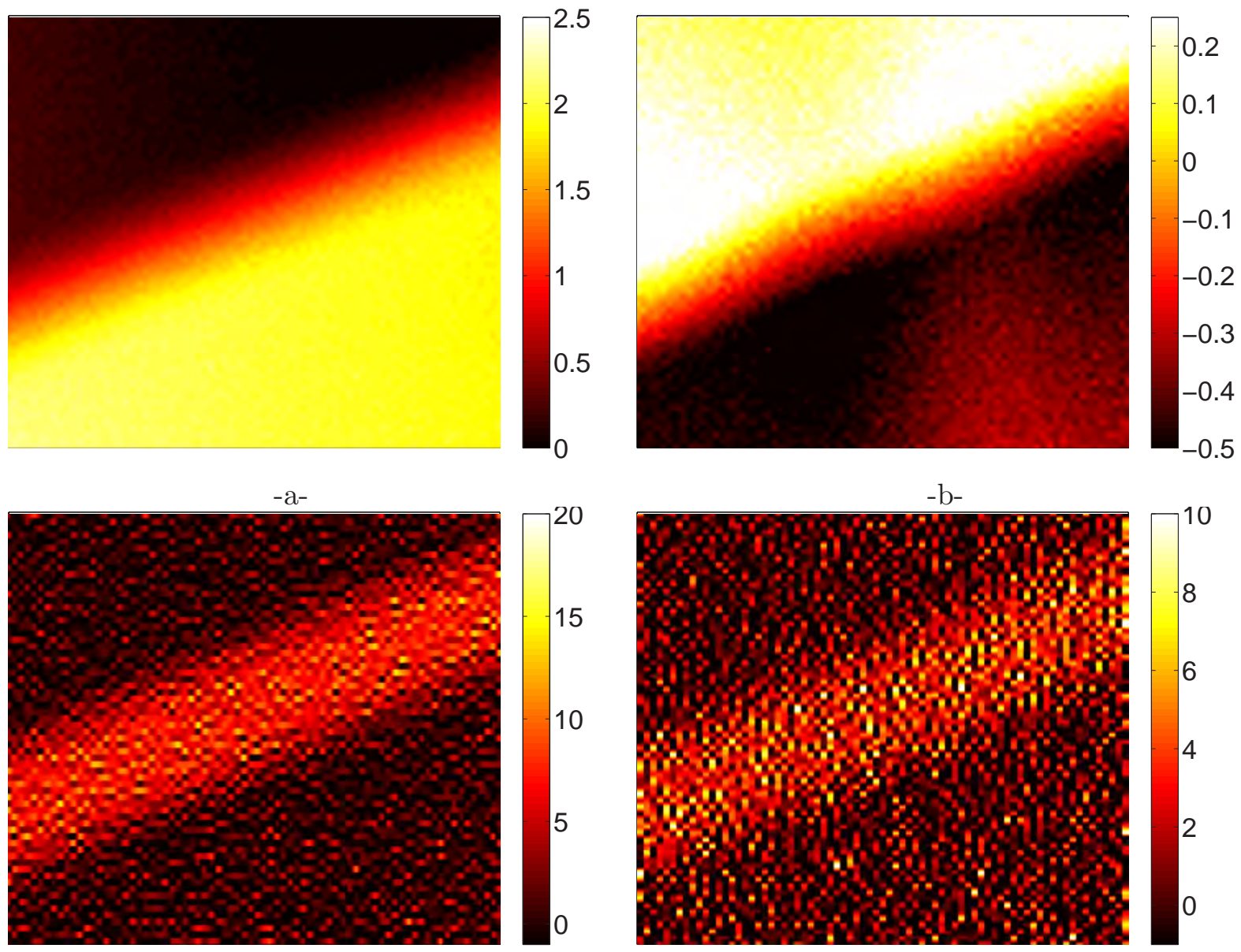

$-\mathrm{C}-$

$-d-$

Figure 12. Vertical $u$ (a) and horizontal $v$ (b) displacement obtained using a standard finite element discretization (Q4) with 16-pixel elements, and the corresponding strain $\epsilon_{u u}(\mathrm{c})$ and $\epsilon_{u v}(\mathrm{~d})\left(\times 10^{-3}\right)$.

In this example, strains (but not displacements) are observed to be discontinuous across the band. This property results presumably from a discontinuity of instantaneous velocities across a boundary that moves over the time lapse separating the two analyzed images. To address this question, Figure 16 shows the strain fields for the previous, the present and the following pairs of images. One observes the propagation of the band from the top of the region of interest. The band moves downward by 308 and 262 pixels, respectively in between successive image pairs. Furthermore, the three consecutive band widths are successively 286, 248 and 226 pixels, and their orientations is estimated to be $64^{\circ}, 65^{\circ}$ and $64^{\circ}$, respectively. This shows that the band maintains its orientation but decelerates progressively.

\section{Conclusion}

A novel approach was developed to determine weakly or strongly discontinuous displacement fields based on the comparison of two digital images. The sought displacement field is decomposed onto a basis of continuous and discontinuous functions using Q4 elements as proposed in standard or extended finite element methods. It therefore allows for a compatibility of the kinematic hypotheses made during the measurement stage and the subsequent identification/validation stages, for instance, by using finite element techniques. Let us note that the discontinuities involved in many DIC techniques are the result of a local kinematic basis (e.g., piecewise-constant translations or strains), and not intended to describe faithfully a genuine discontinuity. Away from the actual discontinuity, the finite element formulation proposed herein has proven to be more robust and accurate (an order of magnitude gain in the uncertainty may be obtained [22]). The key idea of the present algorithms is to preserve this advantage, while allowing 


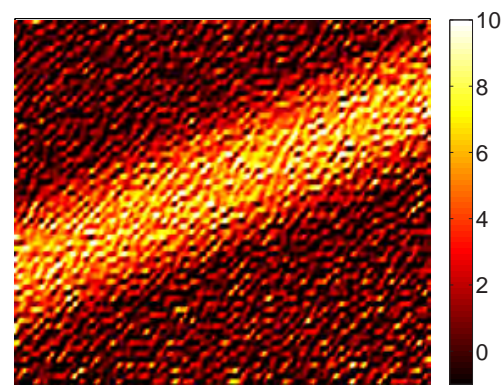

$-a-$

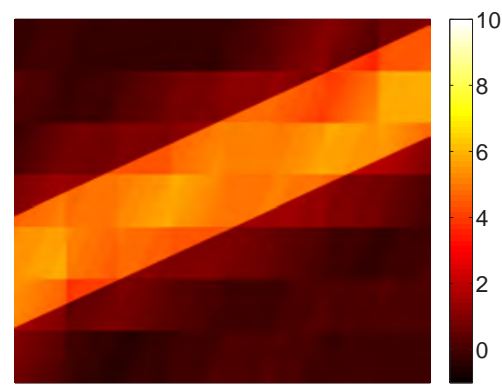

$-\mathrm{C}-$

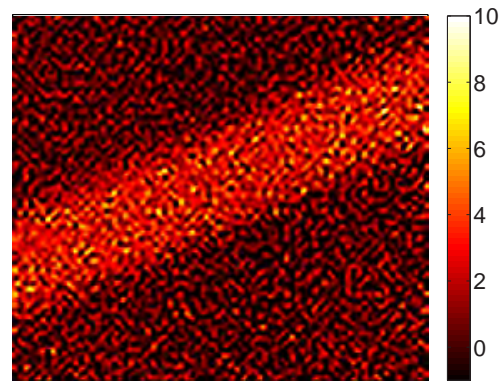

$-b-$

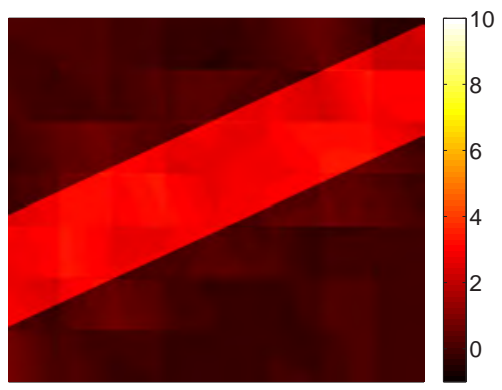

$-\mathrm{d}-$

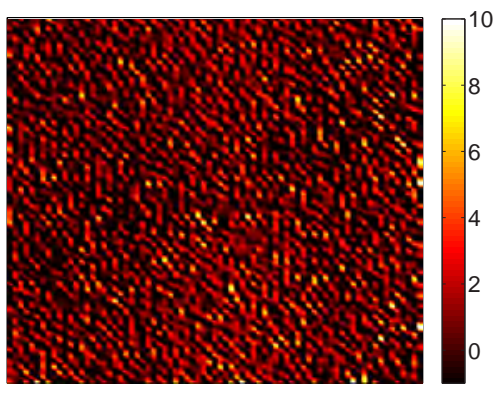

$-\mathrm{c}-$

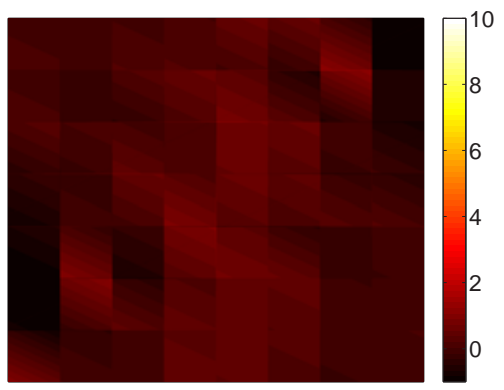

$-\mathrm{e}-$

Figure 13. Normal $\varepsilon_{n n}$, shear $\varepsilon_{t n}$ and tangent $\varepsilon_{t t}$ strain fields $\left(\times 10^{-3}\right)$ obtained using a standard Q4 approach with 16 -pixel elements $(\mathrm{a}, \mathrm{b}, \mathrm{c})$ and the regularized enriched approach (X-Q4r) with 128-pixel elements (d,e,f).

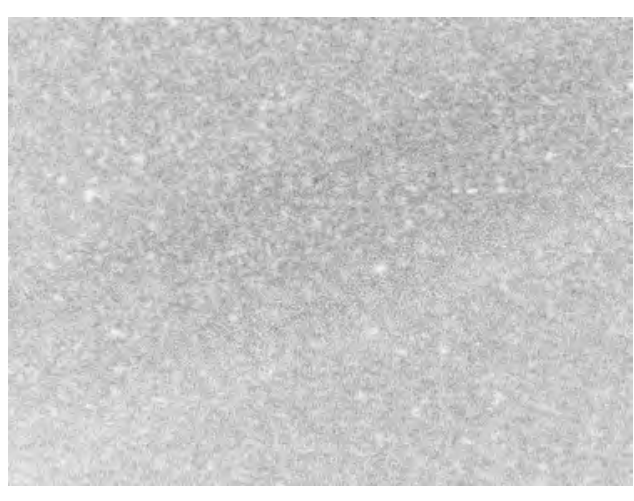

$-a-$
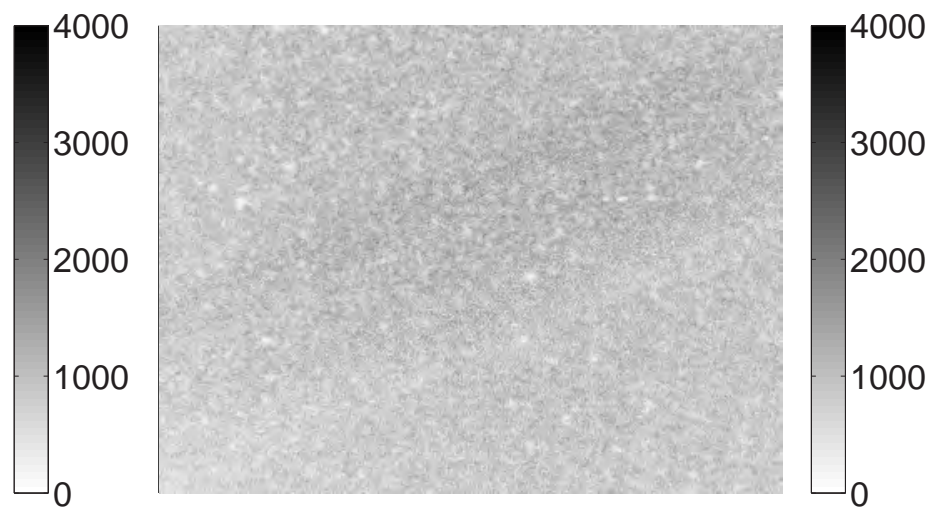

$-\mathrm{b}-$

Figure 14. Error maps for a a standard Q4 approach with 128-pixel elements (a) and the regularized enriched approach (X-Q4r) with 128-pixel elements (b).

for a discontinuity wherever it may happen, irrespective of the mesh, and without additional spurious discontinuities. Furthermore, discontinuities are localized phenomena by nature, then adding enrichments allows for capturing localized phenomena with large element sizes (because away from the discontinuity, large elements with a Q4 kinematics are rich enough to capture the far field), i.e., with lower uncertainty and noise sensitivity levels.

The performance of the algorithm is tested on a reference image to evaluate the reliability of the estimation, which is shown to allow for either an excellent accuracy for homogeneous displacement fields, or for a very well resolved displacement field in the presence of weak and strong discontinuities. Two routes for the evaluation of the performance of a digital image correlation algorithm are proposed. The first is based on the comparison between a prescribed solution and the computed field, and the second evaluates the noise sensitivity. The first allows for an estimate of the accuracy and the second of the stability of the numerical scheme. 


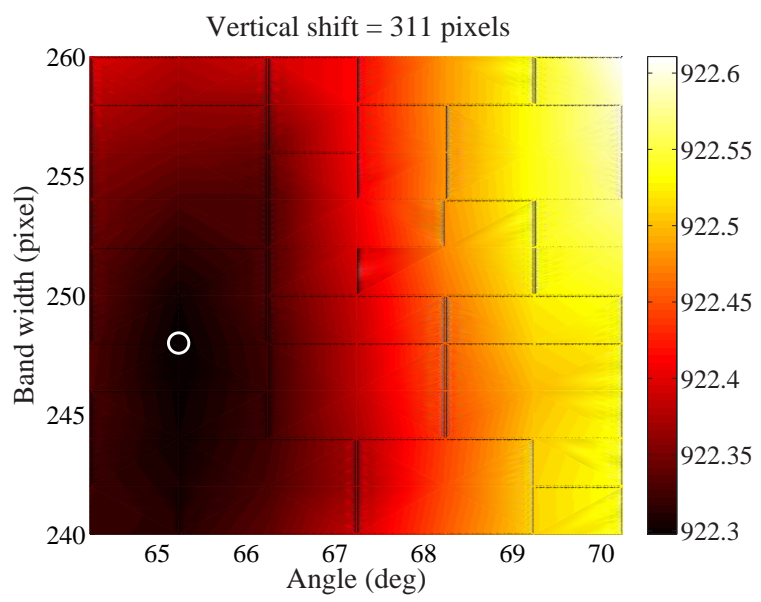

$-a-$

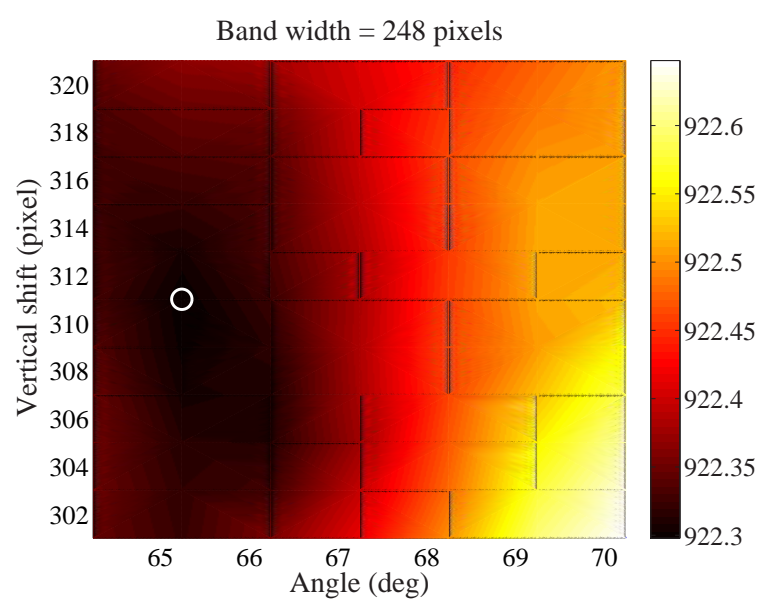

$-b-$

Figure 15. Mean error as a function of the angle and the width of the band for its optimal position (a), as a function of the position and the angle of the band for its optimal width (b). Note that the dynamic of the reference image over the region of interest is 40416 grey levels

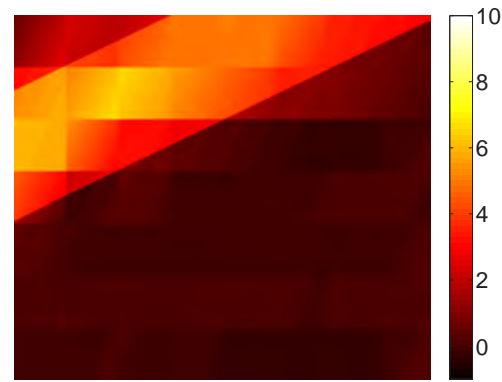

$-\mathrm{a}-$

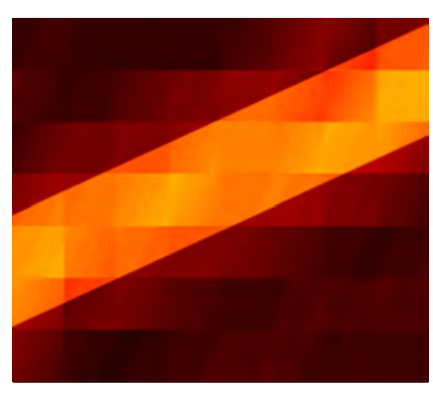

$-\mathrm{C}-$

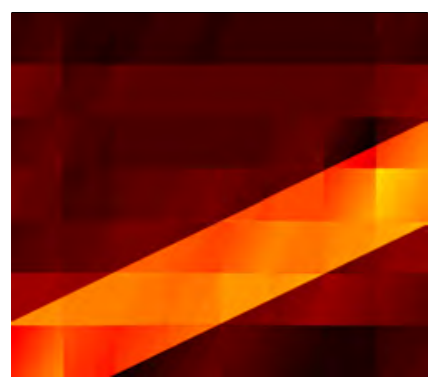

$-\mathrm{f}-$

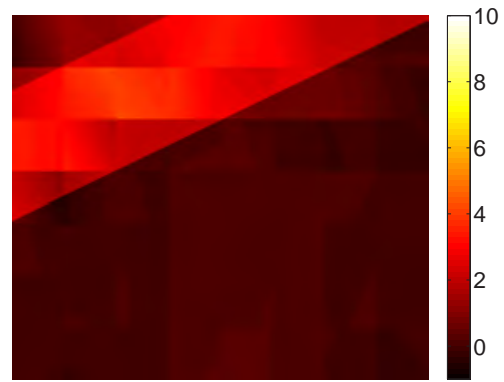

$-b-$

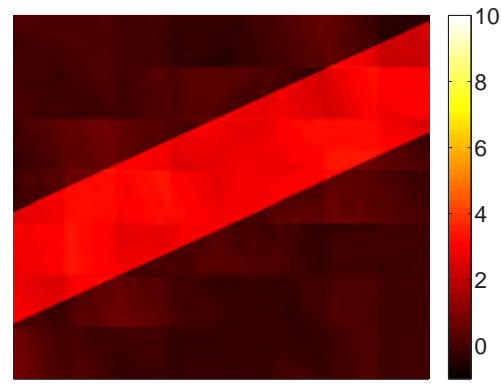

$-\mathrm{d}-$

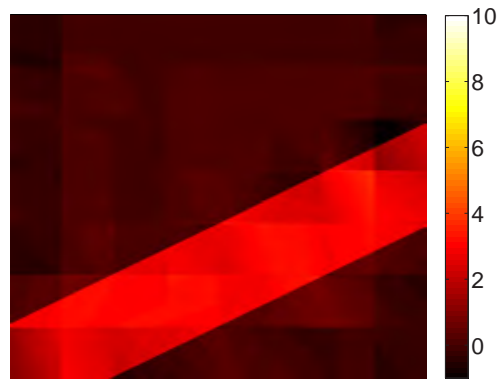

$-g-$

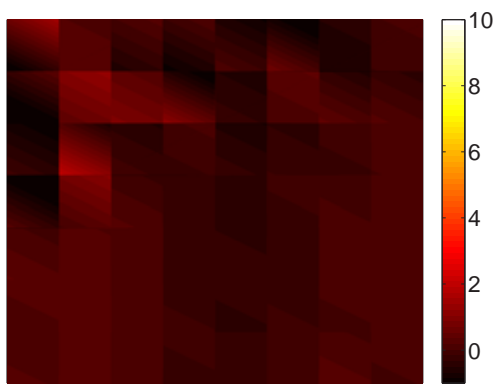

$-\mathrm{C}-$

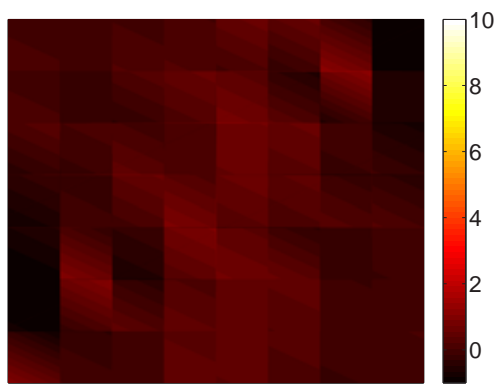

$-\mathrm{e}-$

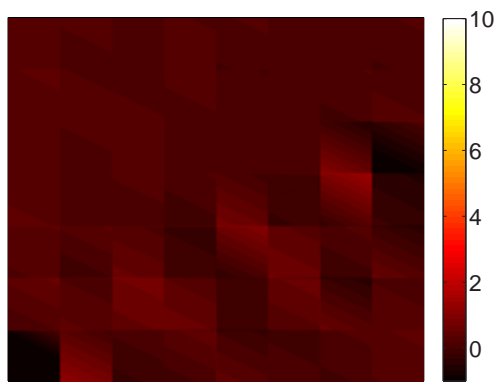

$-\mathrm{h}-$

Figure 16. Normal $\varepsilon_{n n}(\mathrm{a}, \mathrm{c}, \mathrm{f})$, shear $\varepsilon_{t n}(\mathrm{~b}, \mathrm{~d}, \mathrm{~g})$ and tangent $\varepsilon_{t t}(\mathrm{c}, \mathrm{e}, \mathrm{h})$ strain fields $\left(\times 10^{-3}\right)$ obtained using the regularized enriched approach (X-Q4r) with 128-pixel elements for three pairs of images. 
The displacement field is analyzed in the case of shear bands in a concrete-like material. A strong discontinuity is observed, and a discontinuity enrichment allows one to reveal this behavior very accurately since large element sizes can still be used. A Portevin-Le Châtelier band is also studied. In this case, no direct displacement jump is detected. However the strain field appears as discontinuous across the boundaries of the band. The weak discontinuity approach, allowing for larger element sizes, gives access to an incremental strain within the band of the order of $3 \times 10^{-3}$.

Finite-element formulations of image correlation have been shown to benefit from enrichment strategies (e.g., "Embedded Discontinuities," or "Partition of Unity" techniques) in a similar spirit as for modeling purposes. Moreover, noise sensitivity analyses give access quantitatively to a possible poor conditioning, providing simultaneously a guide to control regularization strategies. This remark does not only hold for the displacement or strain discontinuities considered in this paper, but it has a much broader scope of relevance. It points towards the significant gain in accuracy that may be achieved when some knowledge on the specific features of the analyzed displacement field is incorporated in the functional basis of measured displacements.

\section{Acknowledgments}

The authors acknowledge useful discussions with Profs. A. Benallal and J. Lemaitre. This work is part of a project ("PHOTOFIT") funded by the French Agence Nationale de la Recherche.

\section{References}

[1] R. J. Asaro, Acta Metall. 27445 (1979).

[2] J. L., Bassani, Adv. Appl. Mech. 30191 (1994).

[3] S. V. Harren, H. E. Dève and R. J. Asaro, Acta Metall. 362435 (1988).

[4] L. Méric, G. Cailletaud and M. Gasperini, Acta Metall. 42921 (1994).

[5] M. Jirasek, Comp. Meth. App. Mech. Eng. 188307 (2000).

[6] I. Babuska and J. M. Melenk, Int. J. Num. Meth. Eng. 40727 (1997).

[7] N. Moës, J. Dolbow, and T. Belytschko, Int. J. Num. Meth. Eng. 46133 (1999).

[8] G. N. Wells, L. J. Sluys, and R. de Borst, Int. J. Num. Meth. Eng. 531235 (2002).

[9] E. Samaniego and T. Belytschko, Int. J. Num. Meth. Eng. 621857 (2005).

[10] P. M. A. Areias and T. Belytschko, Int. J. Num. Meth. Eng., 66878 (2006).

[11] J. Dolbow and R. Merle, Comp. Mech. 28339 (2001).

[12] N. Moës, M. Cloirec, P. Cartaud, and J. F. Remacle, Comp. Meth. App. Mech. Eng. 1923163 (2003).

[13] L. Allais, M. Bornert, T. Bretheau and D. Caldemaison, Acta Met. Mater. 423865 (1994).

[14] R. Shabadi, S. Kumara, H. J. Roven and E. S. Dwarakadasa, Mat. Sci. Eng. A364 140 (2004).

[15] Y. Berthaud, J.-M. Torrenti and C. Fond, Exp. Mech. 37216 (1997).

[16] J. N. Périé, S. Calloch, C. Cluzel and F. Hild, Exp. Mech. 42318 (2002).

[17] A. Chrysochoos and H. Louche, Int. J. Eng. Sci. 381759 (2000).

[18] H. Louche, P. Vacher and R. Arrieux, Mat. Sci. Eng. A404188 (2005).

[19] N. Ranc and D. Wagner, Mat. Sci. Eng. A394 87 (2005).

[20] D. Thevenet, M. Mliha-Touati and A. Zeghloul, Mat. Sci. Eng. A291 110 (2000).

[21] A. H. Clausen, T. Borvik, O. S. Hopperstad and A. Benallal, Mat. Sci. Eng. A364 260 (2004).

[22] G. Besnard, F. Hild, and S. Roux, Exp. Mech. 46789 (2006).

[23] V. Tarigopula, O. S. Hopperstad, M. Langseth, A. H. Clausen and F. Hild, Int. J. Solids Struct. 45 601 (2008).

[24] S. Roux, F. Hild, P. Viot and D. Bernard, Comp. Part A doi:10.1016/j.compositesa.2007.11.011 (2008).

[25] J. Réthoré, F. Hild, and S. Roux, Int. J. Num. Meth. Eng. 73248 (2008).

[26] J. Réthoré, F. Hild, and S. Roux, Comp. Meth. App. Mech. Eng., 1965016 (2007).

[27] G. Zi and T. Belytschko, Int. J. Num. Meth. Eng., 572221 (2003).

[28] G. Vivier, F. Hild, M. Labrunie, P. Lambert, H. Trumel, Proceedings 17th DYMAT Tech. Meeting, (2007). 